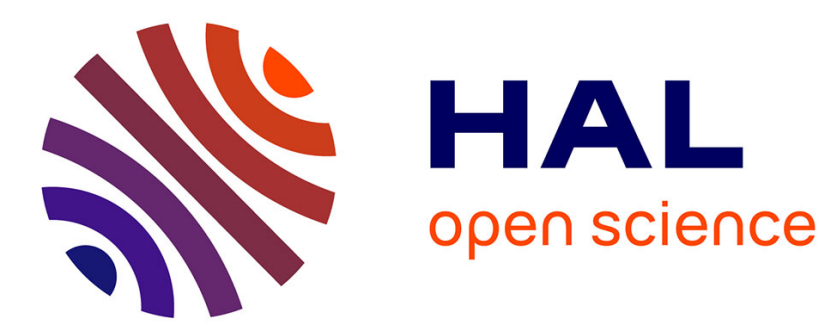

\title{
GEOMETRIC AND KINETIC MODELS OF FRAGMENTATION
}

\author{
X. Campi
}

\section{To cite this version:}

X. Campi. GEOMETRIC AND KINETIC MODELS OF FRAGMENTATION. International Workshop on $\mathrm{MeV}$ and $\mathrm{keV}$ IOns and Cluster Interactions with Surfaces and Materials 2, 1988, Orsay, France. pp.C2-183-C2-188, 10.1051/jphyscol:1989231 。 jpa-00229429

\section{HAL Id: jpa-00229429 https://hal.science/jpa-00229429}

Submitted on 1 Jan 1989

HAL is a multi-disciplinary open access archive for the deposit and dissemination of scientific research documents, whether they are published or not. The documents may come from teaching and research institutions in France or abroad, or from public or private research centers.
L'archive ouverte pluridisciplinaire HAL, est destinée au dépôt et à la diffusion de documents scientifiques de niveau recherche, publiés ou non, émanant des établissements d'enseignement et de recherche français ou étrangers, des laboratoires publics ou privés. 


\section{GEOMETRIC AND KINETIC MODELS OF FRAGMENTATION}

\section{CAMPI}

Division de physique Theorique, Institut de Physique Nucléaire, CNRS LA, F-91406 orsay Cedex, France

Résumé- Nous passons en revue quelques résultats récents de la théorie de la fragmentation. Nous discutons les statistiques qui résultent de différentes constructions géometriques et d'équations cinétiques de fragmentation.

Abstract- We review some recent results on fragmentation theory. We discuss the statistics of various geometric constructions and those of kinetic equations of fragmentation.

Particle fragmentation occurs in a wide range of phenomena in science and technology, including for example the formation of asteroids $/ 1 /$ and stone debris $/ 2 /$, the degradation of polymers $/ 3 /$, the fragmentation of atomic clusters $/ 4,5 /$ and atomic nuclei $/ 6,7 /$. Recently there has been considerable interest in predicting the evolution of the particle-size distribution during fragmentation. This is because it is commonly believed that such distributions contain basic information on the fragmentation process itself, one of the goals being to achieve a classification of the various fragmentation mechanisms. A particularly significant outcome of these studies is the realization that in many circumstances the fragment-size distribution is scale invariant $/ 8,9 /$. Than such a classification would be possible through a scaling formulation. In most of this work solutions have been found for two types of theories: Geometric fragmentation and kinetic fragmentation.

\section{Stat istics of geometric fragmentation}

In the geometric statistical fragmentation one considers the problem of the random partitioning of a line, a surface or a volume, i.e. the processes involving the random positioning of points, lines or surfaces in bodies of dimension $d=1,2$ or 3 respectively. Remark that neither the explicit time dependence nor the detailed breaking mechanism between microscopic constituents is taken into account in these theories. Then deviations of observed fragment-size distribution from those predicted from these statistics would be expected to result from the above simplifications.

In one dimension, the geometric statistics seem to be well defined. For example, consider $n-1$ points of abcissas $x_{1} \ldots x_{n-1}$ uniformely random distributed in a linear chain of lenght $L$ (taking $x_{0}=0$ and $x_{n}=L$ ). The size $I$ of the $n$ fragments is defined as $l_{i}=x_{i}-x_{i-1}(1 \leq i \leq n)$. Than the number of fragments of size $l$ is distributed according to a binomial distribution 


$$
N(1, n)=n(n-1) / L \cdot(1-1 / L)^{n-2}
$$

In a continuous system or when $n, I$ and $L$ goes to infinity, Poisson statistics apply and

$$
N(1, n)=n^{2} / L \exp (-1 n / L)
$$

A different approach is given by percolation theory $/ 10 /$. A linear chain of $L$ points or sites are connected by $L-1$ bonds. Each bond may be either active with a probability $p$ or inactive with a probability $1-p$, the same for all bonds. Points connected by active bonds form a fragment. The number of fragments of size $l$ is given by

$$
N(1, p)=(1-p) p^{1-1}\{2+(L-1-1)(1-p)\}
$$

that reduces to

$$
N(1, p)=L(1-p)^{2} p^{1-1}
$$

for infinitely long chains. We remark that in the first approach (eqs. (1) and (2)) the number of fragments $n$ is fixed, while in percolation theory $p$ is fixed and the number of fragments is a fluctuating quantity, the mean value given by

$$
\langle n(p)\rangle=\Sigma_{l} N(1, p)
$$

For large $L, l$ and $n$ it is easy to prove the equivalence of the two theories. From (4) and (5) one gets

$$
p=1-\langle n(p)\rangle ! L
$$

and inserting in (4) one recovers equation (2).

Experimental tests, in which long thin strips of glass or thin ductile metal rings are broken randomly, show good agreement with distribution (2) in a rather wide range of fragment sizes $/ 11,12 /$. This agreement could support the hypothesis that the broad trend of the distributions is independent of specific material properties and time evolution of the fragmentation process. In more than one dimensions the statistic of geometric fragmentation are much more complex and still many basic points have to be understood. For example, what means uniform random fragmentation of a surface or a volume ?. Mott proposed in $1943 / 13 /$ the forms

$$
\begin{aligned}
& N(a, n) \propto \exp \left(-(2 a n / A)^{1 / 2}\right) \\
& N(v, n) \propto \exp \left(-(3 v n / V)^{1 / 3}\right)
\end{aligned}
$$


for the distributions of fragment areas $a$ and volumes $v$ from an initial area $A$ and volume $V$, respectively. This conjecture was based on the analysis of fragment distribution data from explosively fragmented thick-walled shells, which indicated an exponential distribution with argument proportional to $m^{1 / 3}$, where $m$ was the mass of the fragment. In addition, formulae (7) and (8) appeared to be a natural extension of formula (2) to more dimensions. However this prescription is by far not unique. One can imagine many other uniform random statistics in dimension $d>1$. For example, Grady and Kipp /12/ suggested that the fragment area has to be viewed as a scalar variable. This assumption leads to a fragment distribution similar to (2)

$$
N(a, n) \propto \exp (-a n / A)
$$

The multiplicity of statistics arise from the fact that there are many ways to define random lines on a surface or random surfaces on a volume. Grady and Kipp have studied by computer simulations the fragmentation of a surface by straight lines distributed, oriented or connected at random. In some cases the Mott distribution (7) gives a good description of the observed area distributions while in other cases the linear exponential distribution (9) is definitively better. For the Voronoi construction, both are wrong.

Percolation theory on $d>1$ lattices provides a much richer statistics because it allows for much more complex shapes of the fragments. In addition, the shape of the cluster size distribution depends. drastically on the value of the parameter $p$ defining the probability that bonds be active. When $p$ is close to one most points are connected forming one (and only one) large cluster called percolating cluster. This cluster coexists with very small other clusters. The size distribution of these small clusters is exponential-like and the distribution of all clusters is U-shaped. In the opposite, when $p$ is close to zero most points are isolated or belong to very small clusters. The size distribution is again exponential-like. For large systems a sharply defined percolation threshold $p_{c}$ exists such that for $p<p_{c}$ no percolating cluster exists and for $p>p_{c}$ one percolating cluster exists. At the percolation threshold $p_{c}$ the size distribution is a power law

$$
N(s, p) \propto s^{-\tau}
$$

with a critical exponent $\tau \geq 2$. This behaviour is shown in figure 1 for a cubic lattice containing $5^{3}$ sites. The transition from a non-percolating state to a percolating state is a kind of (second order) phase transition. The percolation transition is a purely geometrical transition in which the clusters are clearly defined static objects.

The structure of the clusters also changes drastically with $p$. Above $p_{c}$ the percolating cluster is a compact object with a rather regular surface. Below $p_{c}$ the clusters have a very ramified structure, much more complex than the ones obtained by the linear constructions of Grady and Kipp.

Percolation ideas have been succesfully applied to fragmentation problems in various contexts /14-16/. 

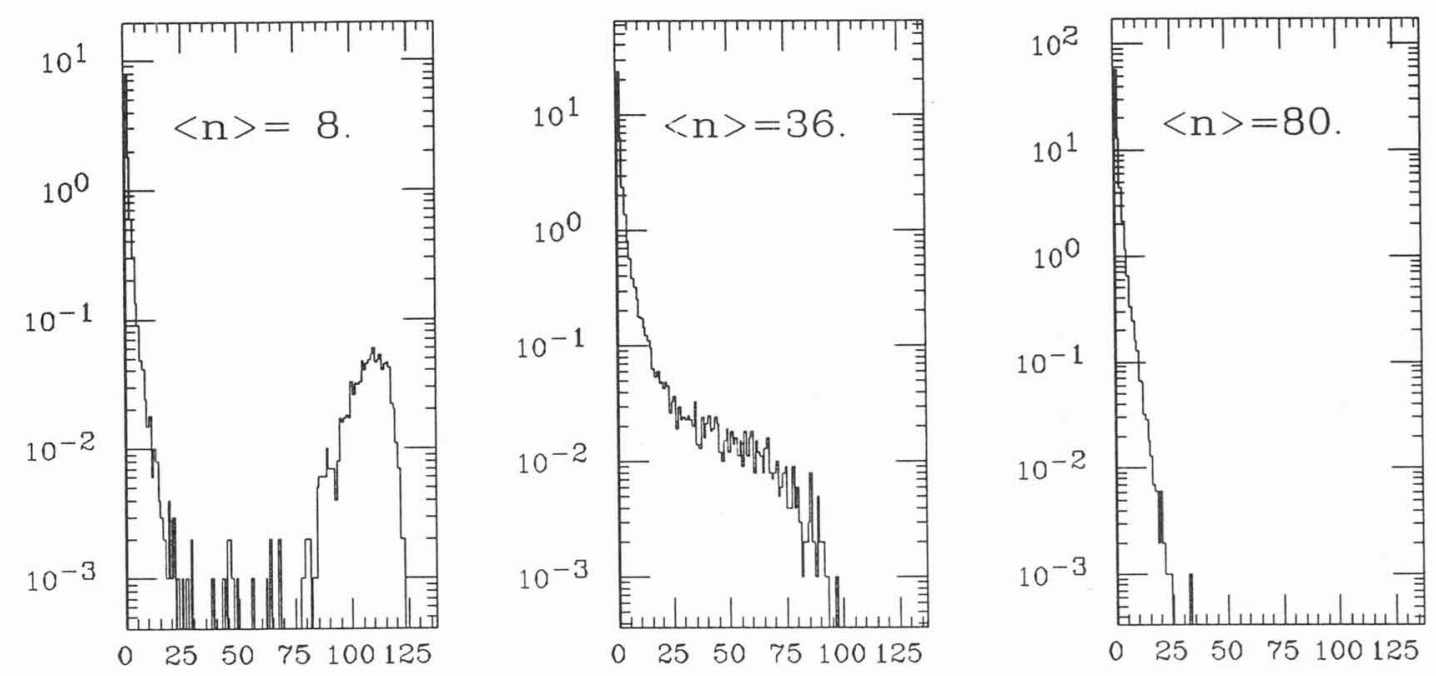

Fig. 1 - Number of clusters $N(s, p)$ of size $s$ in a cubic lattice with $5^{3}$ sites at concentration of active bonds $p=.15$ (right), $p=.30$ (center) and $p=.45$ (left). The mean number of fragments $\langle n\rangle$ is also indicated. Average over 1000 trials.

\section{Statistics of kinetic fragmentation}

So far we have described static approaches of particle fragmentation. In some physical situations it is important to take into account explicitely the time evolution of the fragmentation process. One postulates the validity c of the system of kinetic equations

$$
\frac{\partial}{\partial t} n_{x}(t)=-a(x) n_{x}(t)+\int_{x}^{\infty} n_{y}(t) a(y) f(x \mid y) d y
$$

Here $n_{x}(t)$ is the number of fragments of size $x$ per unit volume at time $t$. The first term in the right-hand side represents the loss of particles of size $x$ due to the breaking into smaller ones at rate $a(x)$. The integral term represents the increase of particles of size $x$ because of the breakup of larger ones. The rate at which $x$ is produced from $y$ is denoted by $f(x \mid y)$. This quantity must be normalized so that mass is conserved

$$
\int_{0}^{y} x f(x \mid y) d x=y
$$

The average number of fragments produced per single fragmentation step is given by

$$
\bar{N}(y)=\int_{0}^{y} f(x \mid y) d x
$$

For the particular case of binary breakup, $f(x \mid y)=f(y-x, y)$ and $\vec{N}=2$.

Exact solutions of equations (11) have been obtained for various homogeneous breakup kernels, i.e. for kernels that satisfy 


$$
\begin{aligned}
& a(x)=x^{\lambda} \\
& f(x \mid y)=y^{-1} b(x / y)
\end{aligned}
$$

For example, for random scission where chains break independently of their lenght and bonds break independently of their position within the chains, than $\lambda=1$ and $b(x / y)=2$. For monodisperse initial conditions $n_{x}(0)=\delta(x-L), L$ being the initial lenght of the chain, the solution is

$$
n_{x}(t)=\exp (-x t)\left[\delta(x-L)+2 t+t^{2}(L-x)\right]
$$

which is as expected equivalent to equation (2).

McGrady and Ziff /17/ have found the general solution for kernels of the form $b(x / y)=(x / y)^{\nu}$, in which the number of particles produced per step is $\overline{\mathrm{N}}=(\nu+2) /(v+1)$, independently of $y$. A "shattering" transition takes place when $\lambda<0$, in which clusters of indefinitely decreasing size are produced, It results a lost of mass to a phase of "zero" size particles. However this is nothing but an artifact due to the continous model. In a discrete version of equations (11), shattering would correspond to a rapid accumulation of monomers. Remark that this process is analogous to but opposite from, the gelation found in aggregating systems, for which the evolution with time of the size distribution resembles that of percolation as a function of the parameter $p$.

In summary, fragment size distributions depend strongly on the fragmentation process. Thus inspecting the experimental data it is possible to gain insight into the physical mechanism of fragmentation of clusters interacting with surfaces and materials.

\section{REFERENCES}

/1/ Dohnanyi, J.S., J.Geophys. Res. 75 (1970) 3468

/2/ Moore, H. J. and Gault, D.E., Ann. Prog. Rep. (Astr. Stud.) U.S.

Geol. Survey Part B (1965) 127

/3/ Johnson, W. R. and Price,C.C. , J. Polym. Sci 45 (1960) 217

/4/ Arnold, M. ,Kowalski, J.,zu Putlitz, G., Stehlin, T. and Trager, F.,Zeit, für Phys, D3 (1986)230

/5/ Katakuse, I., MIcroclusters, eds. Sugano, S. , Nishina, Y. and Ohnishi, Springer-Verlag (Berlin-Heidelberg; 1987)

/6/ Hüfner, J., Phys. Rep. 125 (1985) 130

77 Waddington, C. J. and Freier, P.S., Phys. Rev. C31 (1985) 888

/8/Ziff, R.M. and McGrady, E.D., Macromolecules 19 (1986) 2513

19/ Cheng, Z. and Redner, S., Phys. Rev. Lett. 60 (1988) 2450

110/ Stauffer, D., Introduction to Percolation Theory, Taylor and

Francis, (London and Philadelphia, 1985)

111/ Grady, D.E. and Benson, D. A., Exp. Mech. 23 (1983) 39

/12/ Grady, D.E. and Kipp, M.E., J. Appl. Phys. 58 (1985) 1210 
/13/ Mott, N.F., Ministry of Supply, AC 4035, May, 1945

114/ Englman, R., Jaeger, Z. and Levi, A. Phil. Mag. B50 (1984) 307

/15/ Campi, X., J.Phys. A: Math. Gen. 19 (1986) L917

116/ Campi, X., Phys. Lett. B208 (1988) 208

117./ MeGrady, E.D. and Ziff, M., Phys. Rev. Lett. 58 (1987) 892 